\title{
Vibrational Stark Effect of the Electric-Field Reporter 4-Mercaptobenzonitrile as a Tool for Investigating Electrostatics at Electrode/SAM/Solution Interfaces
}

Gal Schkolnik ${ }^{1}$, Johannes Salewski ${ }^{1}$, Diego Millo ${ }^{1,2}$, Ingo Zebger ${ }^{1}$, Stefan Franzen ${ }^{3}$ and Peter Hildebrandt ${ }^{1, *}$

1 Technische Universität Berlin, Insitut für Chemie, Sekr. PC14, Straße des 17, Juni 135, Berlin, D-10623, Germany; E-Mails: gal.schkolnik@mail.tu-berlin.de (G.S.); johannes.salewski@tu-berlin.de (J.S.); d.millo@vu.nl (D.M.); ingo.zebger@tu-berlin.de (I.Z.)

2 Biomolecular Spectroscopy, LaserLaB Amsterdam, Vrije Universiteit Amsterdam, De Boelelaan 1083, Amsterdam, NL-1081 HV, The Netherlands

3 Department of Chemistry, North Carolina State University, Box 8204, Raleigh, NC 27695, USA; E-Mail: stefan_franzen@ncsu.edu

* Author to whom correspondence should be addressed; E-Mail: hildebrandt@chem.tu-berlin.de; Tel.: +49-0-30-314-21433; Fax: +49-0-30-314-21122.

Received: 9 May 2012; in revised form: 6 June 2012 / Accepted: 11 June 2012 /

Published: 18 June 2012

Abstract: 4-mercaptobenzonitrile (MBN) in self-assembled monolayers (SAMs) on $\mathrm{Au}$ and $\mathrm{Ag}$ electrodes was studied by surface enhanced infrared absorption and Raman spectroscopy, to correlate the nitrile stretching frequency with the local electric field exploiting the vibrational Stark effect (VSE). Using MBN SAMs in different metal/SAM interfaces, we sorted out the main factors controlling the nitrile stretching frequency, which comprise, in addition to external electric fields, the metal-MBN bond, the surface potential, and hydrogen bond interactions. On the basis of the linear relationships between the nitrile stretching and the electrode potential, an electrostatic description of the interfacial potential distribution is presented that allows for determining the electric field strengths on the SAM surface, as well as the effective potential of zero-charge of the SAM-coated metal. Comparing this latter quantity with calculated values derived from literature data, we note a very good agreement for $\mathrm{Au} / \mathrm{MBN}$ but distinct deviations for $\mathrm{Ag} / \mathrm{MBN}$ which may reflect either the approximations and simplifications of the model or the uncertainty in reported structural parameters for $\mathrm{Ag} / \mathrm{MBN}$. The present electrostatic model consistently explains 
the electric field strengths for MBN SAMs on $\mathrm{Ag}$ and $\mathrm{Au}$ as well as for thiophenol and mercaptohexanoic acid SAMs with MBN incorporated as a VSE reporter.

Keywords: interfaces; self-assembled monolayer; electrode; vibrational Stark effect; surface enhanced Raman; surface enhanced infrared; potential of zero-charge

\section{Introduction}

Self-assembled monolayers (SAMs) are widely used in various fields of interfacial and surface science ranging from biosensing and biocatalysis to nano- and microelectronics [1-3]. The outstanding importance of metal/SAM devices has motivated a large number of experimental and theoretical studies of their structural, mechanical, and electronic properties. However, a comprehensive understanding of the interfacial potential distribution in metal/SAM building blocks, particularly in electrochemical environments, has not yet been achieved. One reason for this knowledge gap is the lack of accurate experimental data, which in turn requires appropriate and sufficiently sensitive techniques. In most of the studies, electrochemical methods such as cyclic voltammetry (CV) or electrochemical impedance spectroscopy (EIS) have been employed [4,5], providing information about the charge density at the SAM/solution interface as well as the resistance and the capacity of the monolayer. An alternative and promising approach has been introduced by Oklejas et al. [6,7] who exploited the vibrational Stark effect (VSE) of the nitrile function, attached to amphiphiles that constitute a SAM on an electrode surface. The VSE is defined as the perturbation of vibrational transitions, here the $\mathrm{C} \equiv \mathrm{N}$ stretching mode, by an external electric field. Using surface enhanced Raman (SER) spectroscopy, it was possible to probe the $\mathrm{C} \equiv \mathrm{N}$ stretching frequency of the SAM head groups, thereby providing data that contained information about the interfacial electric field.

A particularly important VSE reporter is 4-mercaptobenzonitrile (MBN; Figure 1), which along with other aromatic nitriles, displays a pronounced sensitivity to the local electric field [8]. Besides its application as a surface modifier, MBN, as well as related molecules, have gained increasing interest as spectral reporters for electric fields in proteins [8-11]. However, regardless of any specific application, it is important to disentangle the various factors controlling the $\mathrm{C} \equiv \mathrm{N}$ stretching frequency, which not only depends on local electric fields but also on the particular environment to which the nitrile is exposed. Particularly, hydrogen bonding interactions have been shown to cause frequency shifts even larger than those induced by changes to the local electric field $[9,12,13]$. Consequently, a profound analysis of the factors influencing the $\mathrm{C} \equiv \mathrm{N}$ stretching mode is a prerequisite for correlating frequency shifts with changes of the local electric field, both at electrode/electrolyte interfaces and on protein surfaces.

Figure 1. Structural formula of 4-mercaptobenzonitrile (MBN).

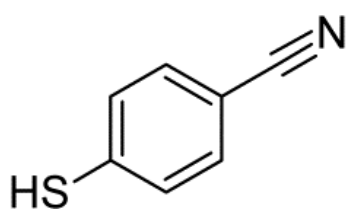


In an electrochemical environment, the analysis of the VSE represents a particular challenge inasmuch as the electric field strength critically depends on interfacial charge distributions, which in turn are related to the potential of zero charge $\left(\mathrm{E}_{\mathrm{pzc}}\right)$. This quantity can readily be determined for bare metal surfaces, but is difficult to assess for SAM-coated metals in aqueous solutions although some attempts have been made [5].

The present study aims at a comprehensive analysis of the VSE of MBN SAMs on Au and Ag electrodes. We have employed surface enhanced infrared absorption (SEIRA) and SER spectroscopy which are the most sensitive techniques for detecting subtle frequency shifts of molecules adsorbed on $\mathrm{Au}$ and $\mathrm{Ag}$ surfaces, respectively. Probing the $\mathrm{C} \equiv \mathrm{N}$ stretching as a function of SAM composition, solvent, and electrode potential, allowed us to disentangle the various factors affecting the nitrile stretching frequency. The results obtained from these experiments constitute the basis for a description of the potential distribution across the electrode/SAM/electrolyte interfaces, thereby refining previous electrostatic models $[4,14,15]$. It is shown that the analysis of VSE can provide information about important electrostatic properties of metal/SAM interfaces such as their potential of zero charge.

\section{Results and Discussion}

\subsection{The Nitrile Stretching Modes of MBN in SAM Coated Ag and Au Surfaces}

Figure 2. $\mathrm{C} \equiv \mathrm{N}$ stretching frequency for $\mathrm{MBN}$ self-assembled monolayers (SAMs) on $\mathrm{Au}$ (black squares) and Ag (red circles), measured by surface enhanced infrared absorption (SEIRA) and surface enhanced Raman (SER) spectroscopy, respectively. The hollow symbols refer to the measurements of the metal/MBN/air interface whereas the solid symbols indicate the data obtained for metal/SAM interfaces in contact with DMSO/buffer solutions of different composition. The aqueous buffer (KPB) consisted of $10 \mathrm{mM}$ potassium phosphate at $\mathrm{pH}=7.0$. The error bars indicate the root-mean square deviations over two measurements (see text for further details).

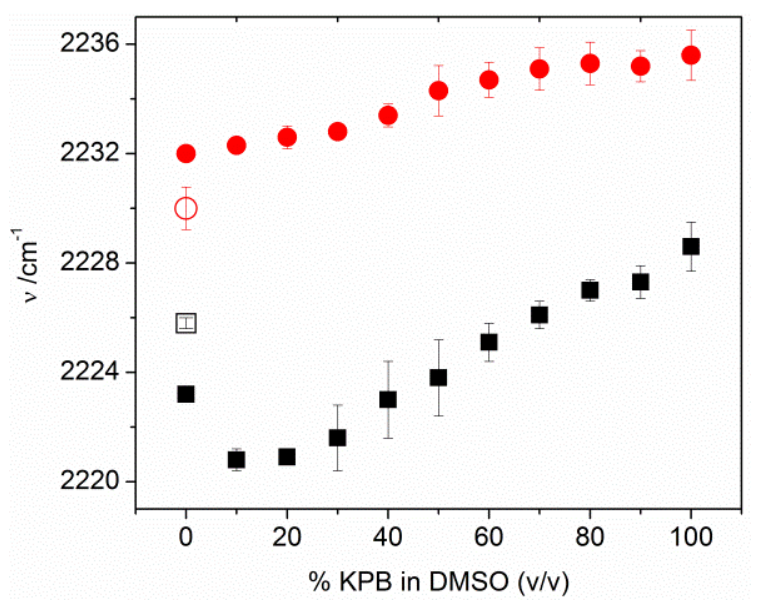

The nitrile stretching frequency of pure MBN SAMs on Ag and Au electrodes, both roughened on the 100-nm scale (see experimental section) was measured by SER and SEIRA spectroscopy, respectively. In the absence of any solvent, i.e., for the $\mathrm{SAM} /$ air interfaces, the $\mathrm{C} \equiv \mathrm{N}$ stretching frequency was determined to be $2230.0 \pm 1.0$ and $2225.8 \pm 0.2 \mathrm{~cm}^{-1}$ for $\mathrm{Ag} / \mathrm{MBN}$ and $\mathrm{Au} / \mathrm{MBN}$, 
respectively (Figure 2). In the case of $\mathrm{Ag} / \mathrm{SAM}$, the frequency increases upon addition of $\mathrm{DMSO}$ /buffer solutions with increasing amounts of aqueous buffer (from 0 to $100 \%$ ) up to a value of $2235.1 \pm 1.0 \mathrm{~cm}^{-1}$ at $100 \%$ aqueous buffer. For Au/SAM in contact with DMSO/buffer solutions, the $\mathrm{C} \equiv \mathrm{N}$ stretching frequency first drops considerably (from $0 \%$ to $10 \%$ buffer) and then steadily increase up to a value $2228.6 \pm 1.0 \mathrm{~cm}^{-1}$ in $100 \%$ buffer solution. This value is essentially the same as that determined also for MBN SAM on Au in $100 \%$ water $\left(2228.4 \pm 1 \mathrm{~cm}^{-1}\right)$.

\subsection{Potential-Dependent Measurements of MBN-SAMs on Ag and Au Surfaces}

Formation of a MBN SAM on Au and Ag electrode surfaces within the electrochemical cell was monitored by CV (Supplementary Information). The nitrile stretching mode of MBN was probed as a function of the electrode potential, which was varied from -0.4 to $+0.6 \mathrm{~V}$ and from -0.5 to $+0.1 \mathrm{~V}$ for $\mathrm{Au} / \mathrm{SAM}$ and $\mathrm{Ag} / \mathrm{SAM}$, respectively. The potential limits were defined by the onset of metal oxidation and reduction of the metal-sulfur bond. A selection of SEIRA and SER spectra is shown in Figure 3. The frequency linearly increases with increasing potential, both for the Au and the Ag electrode. The slope and intercept of the linear fits to the experimental data are similar for the MBN SAMs on Au and Ag (Figure 4). There is no indication of potential-dependent variations in the band profiles. However, in both cases, peak intensity decreases significantly as the potential becomes more positive (Figure 5). This potential-dependent intensity variation has been found to be reversible.

Figure 3. (A) SEIRA spectra of $\mathrm{Au} / \mathrm{MBN}$ and (B) SER spectra of $\mathrm{Ag} / \mathrm{MBN}$, measured at different electrode potentials $E$. Color code: brown: $-0.4 \mathrm{~V}$; pink: $-0.3 \mathrm{~V}$; purple: $-0.2 \mathrm{~V}$; blue: $-0.1 \mathrm{~V}$; black: $0 \mathrm{~V}$; green: $+0.3 \mathrm{~V}$; and red: $+0.6 \mathrm{~V}$.
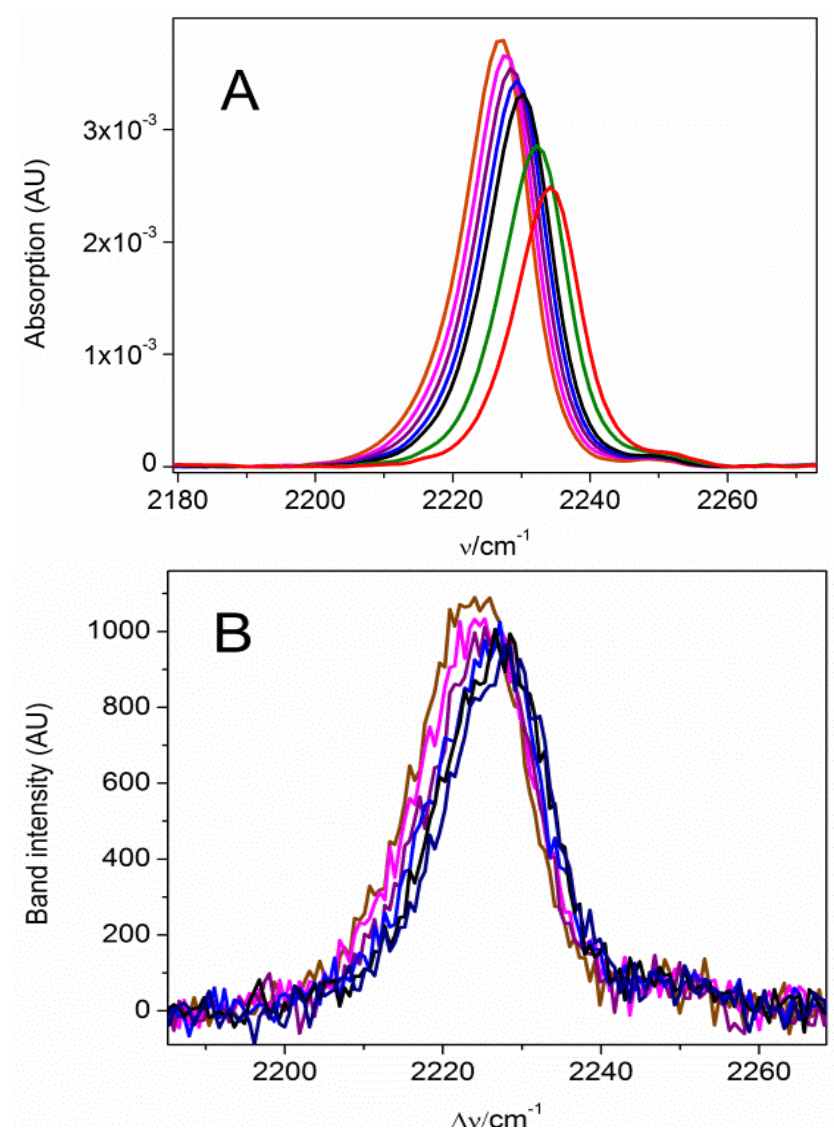
Figure 4. Plots of the nitrile stretching frequency for MBN SAMs on (A) Au and (B) Ag at different electrode potentials measured by SEIRA and SER spectroscopy, respectively. The black squares in (A) and (B) refer to the pure MBN SAMs, whereas the blue circles and red triangles in (A) represent the data for mixed MBN/thiophenol and MBN/mercaptohexanoic acid (MHA) SAMs on Au, respectively. The solid lines are linear fits to the data including the following slopes $m$ and intercepts $b$ : Au/MBN: $m=8.0 \mathrm{~cm}^{-1} / \mathrm{V}^{-1}, b=2230 \mathrm{~cm}^{-1}$; Au/thiophenol/MBN: $m=7.5 \mathrm{~cm}^{-1} / \mathrm{V}^{-1}, b=2229 \mathrm{~cm}^{-1}$; Au/MHA/MBN: $m=8.7 \mathrm{~cm}^{-1} / \mathrm{V}^{-1}$, $b=2228 \mathrm{~cm}^{-1}$; Ag/MBN: $m=8.3 \mathrm{~cm}^{-1} / \mathrm{V}^{-1}, b=2227 \mathrm{~cm}^{-1}$.
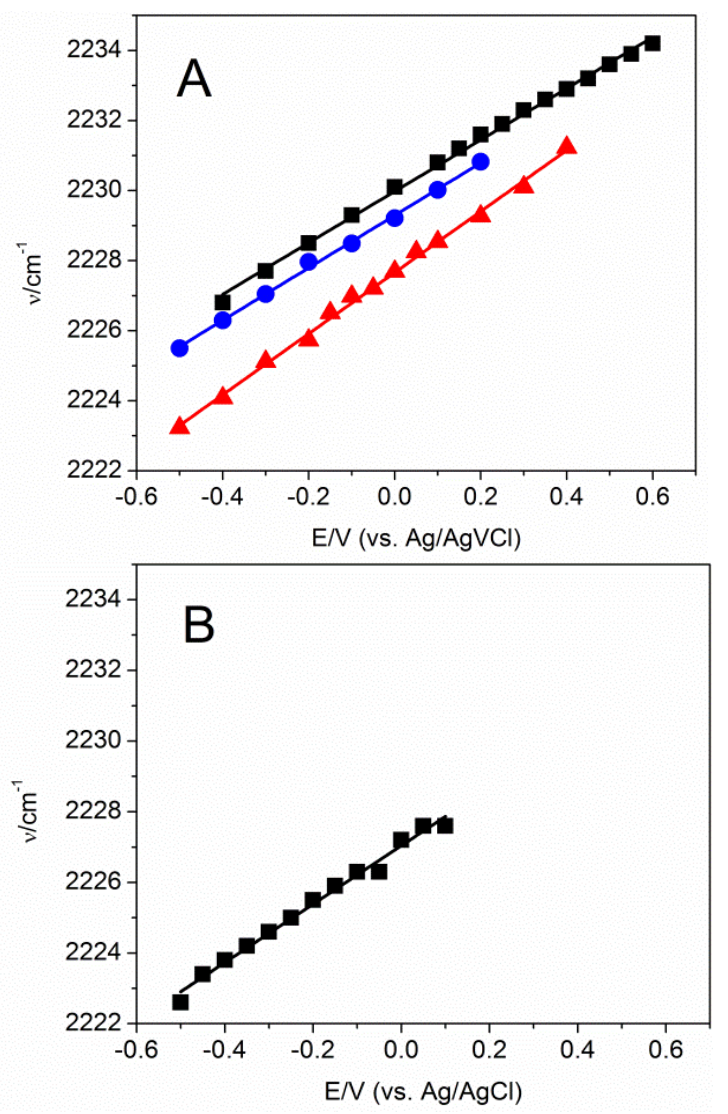

Figure 5. Changes in $\mathrm{C} \equiv \mathrm{N}$ stretching band intensities with the electrode potential for $\mathrm{Au} / \mathrm{MBN}$ (solid black squares), Au/thiophenol/MBN (solid blue circles), and $\mathrm{Au} / \mathrm{MHA} / \mathrm{MBN}$ (solid red triangles) obtained by SEIRA measurements, and Ag/MBN (hollow squares) obtained by SER measurements.

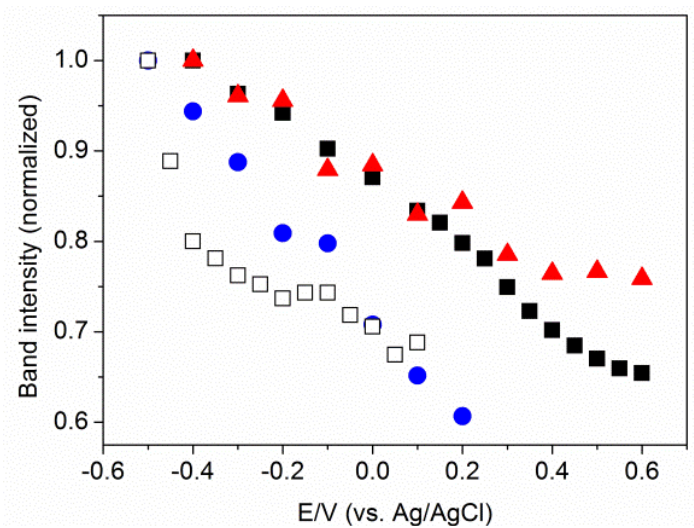




\subsection{MBN in Mixed SAMs on Au Electrodes}

To probe the behavior of the MBN nitrile stretching mode within an environment of charged residues, SEIRA-active Au electrodes were coated with SAMs of mercaptohexanoic acid (MHA). Subsequently, MBN was added to the solution under controlled potential. To integrate MBN into a MHA monolayer, a potential of $-0.4 \mathrm{~V}$ was applied to remove a fraction of MHA molecules from the surface such that binding of MBN from the bulk solution to the vacant adsorption sites became possible. The process was monitored by SEIRA spectroscopy on the basis of the decreasing band intensities for the carboxylate stretching modes of MHA and increasing intensity of the nitrile stretching of MBN (Figure 6). After ca. 12 hours, the SEIRA intensity of the nitrile stretching has reached a value of ca. $4 \%$ of that for the pure $\mathrm{MBN}$ monolayer at the same potential, reflecting the relative contribution of MBN to the composition of the mixed monolayer. Subsequently, potential-dependent SEIRA measurements were carried out to probe the nitrile stretching frequency of MBN within an environment dominated by MHA with protonated and deprotonated head groups. Similar to the pure MBN, the frequency follows a linear relationship with the electrode potential, albeit with slightly different slope and intercept (Figure 4). The larger slope, compared to Au/MBN, may imply that the nitrile groups are less exposed to the aqueous phase [16] and are instead partly buried within the SAM.

Figure 6. SEIRA spectrum of the MHA SAM on Au (A, black trace), measured at open circuit, compared with the SEIRA spectrum obtained after addition of MBN to the solution at an electrode potential of $-0.4 \mathrm{~V}$ ( $\mathbf{B}$, red trace), using spectrum $\mathbf{A}$ as a reference. The negative bands in spectrum $\mathbf{B}$ refer to the removal of MHA from the surface, whereas the positive bands indicate the incorporation of MBN into the SAM.

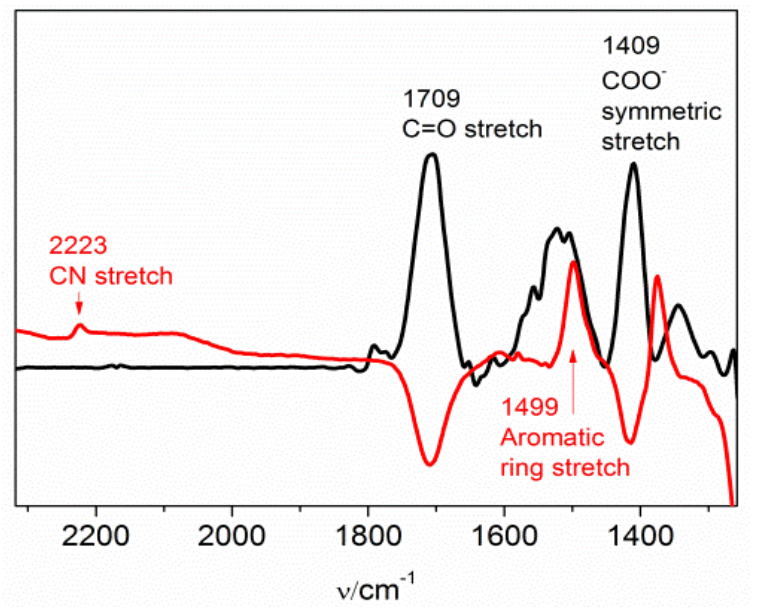

Incorporation of MBN into a thiophenol SAM on an $\mathrm{Au}$ electrode was achieved in a similar way, however, at an electrode potential of $0.0 \mathrm{~V}$. The nitrile stretching was detected by SEIRA spectroscopy. This band remains after removal of excess MBN from the cell and careful rinsing the electrode (Supplementary Information). Detachment of MBN from the electrode was only possible by reductive desorption, i.e., upon applying an electrode potential of $-1.5 \mathrm{~V}$ that led to an irreversible loss of the band. Potential-dependent SEIRA measurements demonstrated again a behavior similar to that of a pure MBN SAM (Figure 4). In this case, however, the slope is lower than for the pure SAM, possibly indicating that in this system the $\mathrm{C} \equiv \mathrm{N}$ groups are more exposed to the solvent [16]. 


\subsection{Factors Controlling the Zero-Field Nitrile Stretching Frequency}

For the analysis of the VSE of MBN we use the linear dependence of the $\mathrm{C} \equiv \mathrm{N}$ stretching frequency $v$ on the electric field $\overrightarrow{E_{F}}$ according to Equation (1):

$$
v=v_{0}-\Delta \vec{\mu} \cdot \overrightarrow{E_{F}}
$$

where $\Delta \vec{\mu}$ is the difference dipole moment (between the ground and excited vibrational states) and $v_{0}$ is the frequency in the absence of an external electric field. Neglecting the higher terms in the electric field dependence is justified a posteriori (vide infra). For MBN and other nitrile-containing VSE labels, $\Delta \vec{\mu}$ has been determined both in frozen 2-methyl-tetrahydrofurane glass and after attachment to a protein $[8,16]$, indicating that this quantity is lowered by about $20 \%$ in aqueous solutions compared to a hydrophobic environment [16]. Accordingly, we have scaled the value of $\Delta \vec{\mu}=7.4 \times 10^{-9} \mathrm{~cm}^{-1} \cdot \mathrm{V}^{-1} \cdot \mathrm{m}$, determined for $\mathrm{MBN}$ in the hydrophobic solvent 2-methyltetrahydrofurane [8], by a factor of 0.8 to obtain an approximate value for MBN SAMs with the nitrile function exposed to the aqueous solution, i.e., $\Delta \vec{\mu}=6.0 \times 10^{-9} \mathrm{~cm}^{-1} \cdot \mathrm{V}^{-1} \cdot \mathrm{m}$. The value for the zero-field frequency $v_{0}$ determined for a protein-attached MBN in an aqueous environment, as well as that determined for MBN in a frozen 2-methyl-tetrahydrofurane glass cannot be adopted for MBN SAMs, as electric field and hydrogen bonding interactions, as well as binding to the different metals and the surface potential of the SAM may affect the $\mathrm{C} \equiv \mathrm{N}$ stretching frequency.

The $\mathrm{Au} / \mathrm{MBN} /$ air and $\mathrm{Ag} / \mathrm{MBN} /$ air systems present $\mathrm{C} \equiv \mathrm{N}$ stretching frequencies at 2225.8 and $2230.0 \mathrm{~cm}^{-1}$, respectively. For comparison, the $\mathrm{C} \equiv \mathrm{N}$ stretching frequency of benzonitrile in the gas phase is observed at $2238 \mathrm{~cm}^{-1}$ [17], indicating a decrease in the $\mathrm{C} \equiv \mathrm{N}$ stretching frequency upon metal binding and SAM formation.

Upon inserting the MBN-coated metal electrodes into a DMSO solution, the polar solvent molecules tend to orient in the SAM/solvent interface. The resulting surface potential causes a downshift of the $\mathrm{C} \equiv \mathrm{N}$ stretching frequency by $2.6 \mathrm{~cm}^{-1}$ in the case of Au. Taking into account the potential-dependence of the $\mathrm{C} \equiv \mathrm{N}$ stretching frequency (Figure 4), a frequency downshift is equivalent to a negative shift of the electrode potential. Accordingly, we conclude that the DMSO molecules are preferentially aligned with the partially negatively charged oxygen atoms facing the SAM surface. Conversely, a $2-\mathrm{cm}^{-1}$ upshift is observed in the case of $\mathrm{Ag} / \mathrm{MBN}$ in contact with $100 \%$ DMSO, pointing to the opposite orientation of the DMSO molecules with respect to the SAM surface.

In addition to the above observations, the response of the $\mathrm{C} \equiv \mathrm{N}$ stretching frequency to the admixture of aqueous buffer is different for $\mathrm{Au} / \mathrm{MBN}$ and $\mathrm{Ag} / \mathrm{MBN}$. The further decrease of the frequency at small amounts of buffer for $\mathrm{Au} / \mathrm{MBN}$ may be attributed to the parallel orientation of DMSO and the even more polar water molecules, both with the oxygen atoms pointing to the SAM, such that surface potential becomes more negative. However, with increasing buffer content, hydrogen bonding interactions of water molecules with the nitrile group gain importance. Such interactions have been shown to exert the opposite effect on the $\mathrm{C} \equiv \mathrm{N}$ stretching frequency $[9,12,13]$ which eventually dominates and thus leads to an increase of the frequency with the buffer content. In contrast, in the Ag-bound MBN SAM the nitrile stretching frequency increases monotonically, since in this case the solvent molecule (DMSO, $\mathrm{H}_{2} \mathrm{O}$ ) orientation and hydrogen bonding affect the nitrile stretching in the same direction. It is interesting to note that the $\mathrm{C} \equiv \mathrm{N}$ stretching frequency of MBN in DMSO solution 
was recorded at $2227.7 \mathrm{~cm}^{-1}$ and the corresponding value for an aqueous solution was estimated to be $2233.6 \mathrm{~cm}^{-1}$ (Supplementary Information). These values lie between the respective frequencies for the $\mathrm{Au}$ - and Ag-bound MBN SAM, consistent with the conclusion that the SAM-coated metal surfaces are oppositely charged at open circuit.

On the basis of these results we may now estimate the zero-field frequency $v_{0}$ of the $\mathrm{Ag} / \mathrm{SAM} / \mathrm{buffer}$ and $\mathrm{Au} / \mathrm{SAM} / \mathrm{buffer}$ systems, i.e., the frequency that includes the effect of metal-sulfur bond formation and the hydrogen bonding interaction in the aqueous solution while excluding the effect of the (open circuit) metal electrode potential and thus that of the surface potential at the SAM/solution interface. This value is experimentally not accessible. However, we may assume that the effect of hydrogen bonding on the $\mathrm{C} \equiv \mathrm{N}$ stretching frequency in an aqueous environment is independent of the existence of the metal-sulfur bond. Correspondingly, the frequency upshift of $7.6 \mathrm{~cm}^{-1}\left(\Delta v_{\mathrm{HB}}\right)$ from solid MBN (2226 $\mathrm{cm}^{-1}$ [11]) to MBN in aqueous solution $\left(2233.6 \mathrm{~cm}^{-1}\right)$ (Supplementary Information) should be approximately the same as the frequency shift arising from the transfer of the $\mathrm{Ag} / \mathrm{MBN} /$ air $\left(2230.0 \mathrm{~cm}^{-1}\right)$ and $\mathrm{Au} / \mathrm{MBN} /$ air $\left(2225.8 \mathrm{~cm}^{-1}\right)$ systems into an aqueous solution in the absence of any effects due to the electrode charge. Correspondingly, the addition of the $\Delta v_{\mathrm{HB}}$ increment of $7.6 \mathrm{~cm}^{-1}$ to the frequencies of the metal/MBN/air interfaces is considered to be a reasonable approximation for the zero-field frequencies and we thus obtain $v_{0}=2237.6 \mathrm{~cm}^{-1}$ and $v_{0}=2233.4 \mathrm{~cm}^{-1}$ for the MBN SAMs on $\mathrm{Ag}$ and $\mathrm{Au}$, respectively.

\subsection{Electrostatic Model}

On the basis of an electrostatic description of the interfacial potential distribution for SAM-coated devices by Smith and White [4] and its further developments [14,15], the electric field at the $\mathrm{SAM} /$ solution interface is given by Equation (2):

$$
\left|\overrightarrow{E_{F}}\right|=\frac{\varepsilon_{S} \kappa \varepsilon_{0}}{\varepsilon_{0}\left(\varepsilon_{C}+\varepsilon_{S} \kappa d_{C}\right)}\left(E-E_{p z c}\right)-\frac{\sigma_{C}}{\varepsilon_{0}\left(\varepsilon_{C}+\varepsilon_{S} \kappa d_{C}\right)}
$$

where $\varepsilon_{\mathrm{c}}$ and $\varepsilon_{\mathrm{s}}$ are the dielectric constants of the SAM and aqueous solution, respectively; $\mathrm{d}_{\mathrm{c}}$ is the thickness of the MBN monolayer, $\varepsilon_{0}$ denotes the permittivity, and $\kappa$ is the reciprocal Debye length. The term $\sigma_{\mathrm{c}}$ refers to the charge density on the SAM surface which for carboxyl-terminated SAMs has been related to apparent $\mathrm{pK}_{\mathrm{a}}$ of the head groups. In a more general sense, $\sigma_{\mathrm{c}}$ may be regarded as the charge density in the inner Helmholtz layer reflecting the contributions of charged SAM head groups as well as those of the ions and water dipoles aligned in the immediate vicinity of the SAM surface. This surface charge density, originally assumed to be constant, varies with the electrode potential E, or more precisely, with the difference between the electrode potential $\mathrm{E}$ and the potential of zero charge of the metal $\mathrm{E}_{\mathrm{pzc}}$. Here, we assume in a first approximation a linear relationship according to Equation (3):

$$
\sigma_{C}=\sigma_{0}+k\left(E-E_{p z c}\right)
$$

where $k$ is a constant and $\sigma_{0}$ is the charge density at $E_{\mathrm{pzc}}$. Equation (3) may be combined with Equation (2) to afford Equation (4)

$$
\left|\overrightarrow{E_{F}}\right|=\frac{\varepsilon_{S} \kappa \varepsilon_{0}}{\varepsilon_{0}\left(\varepsilon_{C}+\varepsilon_{S} \kappa d_{C}\right)}\left(E-E_{p z c}\right)-\frac{\sigma_{0}}{\varepsilon_{0}\left(\varepsilon_{C}+\varepsilon_{S} \kappa d_{C}\right)}-\frac{k\left(E-E_{p z c}\right)}{\varepsilon_{0}\left(\varepsilon_{C}+\varepsilon_{S} \kappa d_{C}\right)}
$$

and after rearrangement: 


$$
\left|\overrightarrow{E_{F}}\right|=\frac{\varepsilon_{S} \kappa \varepsilon_{0}-k}{\varepsilon_{0}\left(\varepsilon_{C}+\varepsilon_{S} \kappa d_{C}\right)}\left(E-E_{p z c}\right)-\frac{\sigma_{0}}{\varepsilon_{0}\left(\varepsilon_{C}+\varepsilon_{S} \kappa d_{C}\right)}
$$

Note that Equation (5) indicates a linear relationship between the electrode potential and the electric field which, taking into account the observed linear correlation between the nitrile stretching frequency and $E$ (Figure 4), justifies neglecting higher order terms in the analysis of the VSE (cf. Equation (1)). For the electric-field dependence of the $\mathrm{C} \equiv \mathrm{N}$ stretching frequency, we have to take into account the definition of the nitrile dipole axis pointing from the carbon to the nitrogen such that $\Delta \vec{\mu}$ has a negative sign [18]. Thus combining Equation (5) and Equation (1) affords:

$$
v=v_{0}+|\Delta \vec{\mu}| \cos \theta\left(\frac{\varepsilon_{S} \kappa \varepsilon_{0}-k}{\varepsilon_{0}\left(\varepsilon_{C}+\varepsilon_{S} \kappa d_{C}\right)}\left(E-E_{p z c}\right)-\frac{\sigma_{0}}{\varepsilon_{0}\left(\varepsilon_{C}+\varepsilon_{S} \kappa d_{C}\right)}\right)
$$

where $\theta$ refers to the angle formed by the vector of the nitrile dipole moment and that of the electric field, arising from the scalar multiplication (see Equation (1)). Rearranging Equation (6) leads to:

$$
v=v_{0}-|\Delta \vec{\mu}| \cos \theta\left(\frac{\left(\varepsilon_{S} \kappa \varepsilon_{0}-k\right) E_{p z c}+\sigma_{0}}{\varepsilon_{0}\left(\varepsilon_{C}+\varepsilon_{S} \kappa d_{C}\right)}\right)+|\Delta \vec{\mu}| \cos \theta\left(\frac{\varepsilon_{S} \kappa \varepsilon_{0}-k}{\varepsilon_{0}\left(\varepsilon_{C}+\varepsilon_{S} \kappa d_{C}\right)}\right) E
$$

where the first two terms on the right hand side of Equation (7) account for the intercept $(b)$ of the linear fits in Figure 4, and the last term corresponds to the slope $(m)$. From the slope term one may

\begin{tabular}{|c|c|c|c|c|c|c|c|}
\hline & $\begin{array}{c}\text { Slope }^{b} \\
\left(\mathrm{~cm}^{-1} / \mathrm{V}\right)\end{array}$ & $\begin{array}{c}\text { Intercept }^{\text {b }} \\
\left(\mathrm{cm}^{-1}\right)\end{array}$ & $\begin{array}{c}k^{c} \\
\left(C V^{-1} m^{-2}\right) \\
\end{array}$ & $\begin{array}{c}\mathbf{E}_{0}, \text { exp. }^{\mathbf{c}} \\
(\mathrm{V})\end{array}$ & $\begin{array}{c}\sigma_{0}{ }^{\mathrm{c}} \\
\left(\mathrm{Cm}^{-2}\right)\end{array}$ & $\begin{array}{c}E_{0} \text {, } \text { calc. }^{d} \\
(V)\end{array}$ & $\begin{array}{c}\mathrm{E}_{0} \text {, calc. }^{\mathrm{e}} \\
(\mathrm{V})\end{array}$ \\
\hline $\mathrm{Ag} / \mathrm{MBN}$ & 8.3 & 2227 & -0.117 & 1.277 & 1.005 & 1.685 & 1.936 \\
\hline $\mathrm{Au} / \mathrm{MBN}$ & 8.0 & 2230 & -0.186 & 0.425 & 0.199 & 0.215 & 0.402 \\
\hline $\mathrm{Au} / \mathrm{TP}^{\mathrm{f}} / \mathrm{MBN}$ & 7.5 & 2229 & -0.153 & 0.587 & 0.267 & - & - \\
\hline $\mathrm{Au} / \mathrm{MHA} / \mathrm{MBN}$ & 8.7 & 2228 & -0.232 & 0.621 & 0.330 & - & - \\
\hline \multicolumn{8}{|c|}{$\begin{array}{l}{ }^{a} \text { calculated according to Equation (7), using } \varepsilon_{\mathrm{S}}=78, \kappa=5 \cdot 10^{8} \mathrm{~m}^{-1}, \varepsilon_{0}=8.854 \times 10^{-12} \mathrm{C} \cdot \mathrm{V}^{-1} \cdot \mathrm{m}^{-1} \text {, } \\
\varepsilon_{\mathrm{c}}=3[19], \Delta \mu=6.0 \times 10^{-9} \mathrm{~cm}^{-1} \cdot \mathrm{V}^{-1} \cdot \mathrm{m} \text {, and } \mathrm{d}_{\mathrm{c}}=6.8 \times 10^{-10} \mathrm{~m} \text { for Au} / \mathrm{MBN} \text { and } 8.9 \times 10^{-10} \mathrm{~m} \text { for Ag} / \mathrm{MBN} \\
\text { as described in the text. For } \mathrm{E}_{\mathrm{pzc}} \text {, we have used }-0.92 \mathrm{~V} \text { and }+0.06 \mathrm{~V} \text { for of polycrystalline Ag and Au, } \\
\text { respectively [20,21]; }{ }^{\mathrm{b}} \text { taken from the linear fits in Figure } 4 ;{ }^{\mathrm{c}} \text { as defined by Equation (3), derived from the } \\
\text { experimental data according to Equations (7) and (8); }{ }^{\mathrm{d}} \text { calculated using Equation (11), according to Wang et } \\
\text { al. }[22] ;{ }^{\mathrm{e}} \text { calculated using Equations }(11-13) \text {, according to Heimel } \text { et al. }[23] ;{ }^{\mathrm{f}} \mathrm{TP} \text { : thiophenol. }\end{array}$} \\
\hline
\end{tabular}
determine the quantity $\mathrm{k}$ for the various metal/SAM interfaces (Table 1).

Table 1. Electrostatic parameters for various metal/MBN interfaces ${ }^{\text {a }}$.

For these calculations, estimates for the tilt angle of the nitrile group are required. For $\mathrm{MBN}$ on $\mathrm{Au}$ $\theta$ was determined to be $\sim 40^{\circ}$ [24], which is similar to that for a thiophenol monolayer on $\mathrm{Au}$ $\left(49^{\circ}-54^{\circ}\right)[25,26]$. Reported angles for MBN and thiophenol on Ag, however, differ significantly, i.e., $24^{\circ}-28^{\circ}$ for thiophenol on $\mathrm{Ag}$ [27,28], and, most surprisingly, ca. $0^{\circ}$ for MBN [29]. In view of this striking discrepancy between thiophenol and $\mathrm{MBN}$ on $\mathrm{Ag}$, which is in sharp contrast to the findings for $\mathrm{Au}$, one has to consider the reported value for $\mathrm{Ag} / \mathrm{MBN}$ with caution. Nevertheless, for the present calculations we first adopt $49^{\circ}$ and $0^{\circ}$ for $\mathrm{Au} / \mathrm{MBN}$ and $\mathrm{Ag} / \mathrm{MBN}$, respectively. These values are also included in determining the thickness of the SAM, $\mathrm{d}_{\mathrm{c}}$, using an Ag-S bond length of $2.4 \AA$ and Au-S bond length of $1.9 \AA$ [30,31], a sulfur-nitrogen distance of $6.5 \AA$ as well as the respective values of $\theta$ 
discussed above. As a result, $\mathrm{d}_{\mathrm{c}}$ is evaluated to be $6.8 \times 10^{-10} \mathrm{~m}$ and $8.9 \times 10^{-10} \mathrm{~m}$ for $\mathrm{Au} / \mathrm{MBN}$ and $\mathrm{Ag} / \mathrm{MBN}$, respectively.

Now we consider the potential at which the electric field at the position of the nitrile group is zero (Equation (5)). This potential may be considered as the effective potential of zero-charge $\mathrm{E}_{0}$ for the entire metal/SAM/solution system. Expressing Equation (7) for $E=E_{0}$, in terms of the slope m and intercept $b$ of the linear fits to the experimental data in Figure 4, we then obtain

$$
E_{0}=\frac{v_{0}-b}{m}
$$

Note that $E_{0}$ may, therefore, differ substantially from $E_{\mathrm{pzc}}$, which refers to the potential of zero-charge of the bare metal. These experimentally determined $E_{0}$ values are listed in Table 1 . We further use these $E_{0}$ values obtained in this way to calculate the charge densities in the inner Helmholtz layer at zero-field $\left(\sigma_{0}\right)$ according to Equation (9):

$$
\left(E_{0}-E_{p z c}\right)\left(\varepsilon_{S} \kappa \varepsilon_{0}-k\right)=\sigma_{0}
$$

which is derived from Equation (7) when $\mathrm{E}=\mathrm{E}_{0}$ and $v=v_{0}$.

Equation (5) may now be used to calculate the variation of the electric field strength at the SAM surface, i.e., at the position of the nitrile group, as a function of the electrode potential (Figure 7). For all metal/SAM devices, the modulus of the field strength increases with decreasing potential, covering a range from $0.9 \times 10^{8} \mathrm{~V} / \mathrm{m}$ to $-2.5 \times 10^{9} \mathrm{~V} / \mathrm{m}$. Whereas similar values are obtained for $\mathrm{Au} / \mathrm{MBN}$ and $\mathrm{Au} /$ thiophenol/MBN, the modulus of the field strength is larger in the mixed MHA/MBN SAM on Au.

Figure 7. Plots of the electric field strength as a function of the electrode potential, calculated according to Equations (1) and (5), using the experimentally determined $\mathrm{C} \equiv \mathrm{N}$ stretching frequencies for $\mathrm{Au} / \mathrm{MBN}$ (solid black squares), Au/thiophenol/MBN (solid blue circles), $\mathrm{Au} / \mathrm{MHA} / \mathrm{MBN}$ (solid red triangles), and Ag/MBN (hollow squares).

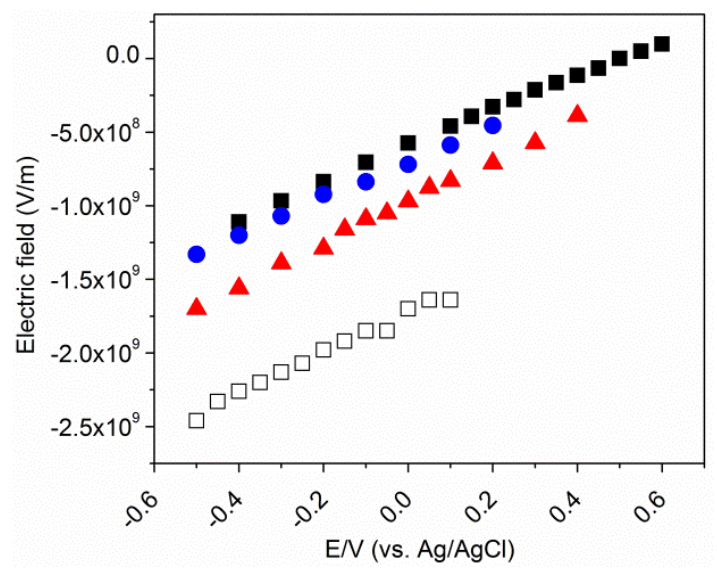

As judged from the SEIRA intensity, this mixed SAM includes only $4 \% \mathrm{MBN}$, however, it is not known if the incorporation of MBN into a preformed MHA SAM leads to a largely homogeneous MBN distribution or to MBN islands within the MHA SAM. In the latter case, one would expect an inhomogeneity of the SEIRA bands, given that there are different electric field strengths at the surface of the dominant MHA part of the SAM and the much smaller MBN islands, leading to different nitrile stretching frequencies in the interior of the MBN island compared to the MBN/MHA boundary. Such 
inhomogeneities are not observed in the SEIRA spectra of $\mathrm{Au} / \mathrm{MHA} / \mathrm{MBN}$, implying that the electric field at the SAM surface does not display significant gradients parallel to the surface or that the distribution of MBN within the MHA SAM is largely homogeneous. In any case, we may conclude that (i) MBN probes the electric field on the MHA SAM surface, and (ii) the magnitude of the electric field on a MHA SAM on $\mathrm{Au}$ at $\mathrm{pH} 7.0$ is only larger by ca. $15 \%$ compared to $\mathrm{Au} / \mathrm{MBN}$, despite the partial dissociation of the carboxylate head groups at $\mathrm{pH} 7.0[5,14,32,33]$. This conclusion is consistent with the finding that the magnitude of the electric field on $\mathrm{Ag} / \mathrm{MBN}$, which is ca. twice as large as that found for $\mathrm{Au} / \mathrm{MBN}$, is quite similar to that estimated for Ag/MHA monolayers [14].

\subsection{The Effective Potential of Zero Charge}

The quantity $E_{0}$ derived from the experiments reflects the change in the potential of zero charge of the pure metal $\left(E_{\mathrm{pzc}}\right)$ due to binding of the dipolar SAM to the metal surface. The effective potential of zero charge $E_{0}$ can also be estimated by calculating the change in the work function of the metal $(\Delta \Phi)$ due to the coating by the SAM. In one approach, suggested by Wang et al. [22], the only parameters affecting $\Delta \Phi$ are assumed to be the direction of the dipole moment of the SAM and its angle with respect to the surface normal $(\beta)$, which can be evaluated from the tilt angle $\theta$ for the nitrile function, taken to be $49^{\circ}$ and $0^{\circ}$ for $\mathrm{Au} / \mathrm{MBN}$ and $\mathrm{Ag} / \mathrm{MBN}$, respectively (vide supra). On the basis of the $\Delta \Phi v s . \cos (\beta)$ relationship determined by Wang et al. [22], one then obtains $\Delta \Phi=0.15$ and $2.6 \mathrm{eV}$ for $\mathrm{Au} / \mathrm{MBN}$ and $\mathrm{Ag} / \mathrm{MBN}$ respectively.

For bare metals, the work function $\Phi_{M}$ is related to the potential of zero charge according to:

$$
E_{p z c}=\Phi_{M}-K
$$

where here $\mathrm{E}_{\mathrm{pzc}}$ is expressed in $\mathrm{V} v s$. NHE and $K$ is a constant that was determined to be 5.01 and $4.61 \mathrm{eV}$ for $\mathrm{Ag}$ and $\mathrm{Au}$, respectively [34,20]. Recommended values for the work function of bare polycrystalline $\mathrm{Ag}$ and $\mathrm{Au}$ are 4.3 and $4.88 \mathrm{eV}[21,35]$ such that one obtains $-0.71 \mathrm{~V}$ and $+0.27 \mathrm{~V}$ for $E_{\mathrm{pzc}}$ of polycrystalline $\mathrm{Ag}$ and $\mathrm{Au}$, respectively. Correspondingly, Equation (10) allows evaluating the effective potential of zero charge from the work function of the SAM-coated metals $\Phi_{\mathrm{M} / \mathrm{SAM}}$ according to Equation (11):

$$
E_{0}=\Phi_{M / S A M}-K=\Phi_{M}+\Delta \Phi-K
$$

such that one obtains, after translating the reference potential scale from $\mathrm{NHE}$ to $\mathrm{Ag} / \mathrm{AgCl}, \mathrm{E}_{0}=0.215$ and $1.658 \mathrm{~V}$ for $\mathrm{Au} / \mathrm{MBN}$ and $\mathrm{Ag} / \mathrm{MBN}$, respectively. The values are in reasonable agreement with the data derived from the VSE analysis (Table 1), specifically in view of the underlying approximations of both the electrostatic model and the $\mathrm{E}_{0}$ calculations.

An alternative for calculating $\Delta \Phi$, suggested by Heimel et al. [23,36], includes the metal-sulfur bond dipole, $\mathrm{BD}$, and the potential difference across the dipolar SAM, $\Delta V_{\text {vac }}$, according to Equation (12):

$$
\Delta \Phi=\Delta V_{v a c}+B D
$$

here $\Delta \mathrm{V}_{\mathrm{vac}}$ is given by Equation (13):

$$
\Delta V_{v a c}=-\frac{\mu_{\perp}}{\epsilon_{0} \epsilon_{e f f} A}
$$


where $\mu_{\perp}$ is the dipole moment component of the SAM in the direction of the surface normal, $A$ is the area of the unit cell, and $\epsilon_{e f f}$ is a correction coefficient suggested to be 2.3 for a SAM of 4-mercaptobiphenylnitrile [23]. Estimating $\mu_{\perp}$, even if $\beta$ is known, is not straightforward as it differs from the mere projection of the single MBN molecular dipole onto the surface normal, due to the depolarization of the MBN dipole by neighboring molecules in the SAM [37]. According to Natan et al. [37], we adopt a value of 3.6 Debye for the dipole moment of the MBN SAM, which is then multiplied by $\cos (\beta)$ to afford $\mu_{\perp}$. On the basis of calculated values for a series of related systems [23], BD is taken to be $-1.05 \mathrm{eV}$ and $-0.27 \mathrm{eV}$ for $\mathrm{Au} / \mathrm{MBN}$ and $\mathrm{Ag} / \mathrm{MBN}$, respectively. The difference between these two values is in good agreement with experimental data reported by Alloway et al. [38]. Thus, one obtains $0.3 \mathrm{eV}(\mathrm{Au} / \mathrm{MBN})$ and $2.9 \mathrm{eV}(\mathrm{Ag} / \mathrm{MBN})$ for $\Delta \Phi$ and eventually $E_{0}=0.402$ and $1.936 \mathrm{~V}$ vs. $\mathrm{Ag} / \mathrm{AgCl}$ for $\mathrm{Au} / \mathrm{MBN}$ and $\mathrm{Ag} / \mathrm{MBN}$, respectively. Here, $E_{0}$ for $\mathrm{Au} / \mathrm{MBN}$ is much closer to the experimentally derived value (Table 1) than in the previous approach, while there is a stronger deviation from $\mathrm{E}_{0}$ for $\mathrm{Ag} / \mathrm{MBN}$. The failure to reproduce the experimentally derived value for $\mathrm{Ag} / \mathrm{MBN}$ in a satisfactory manner does not necessarily argue against the approach proposed by Heimel et al. [23]. In view of the very good agreement for $\mathrm{Au} / \mathrm{MBN}$, one may also question the most uncertain parameter in the calculation for $\mathrm{Ag} / \mathrm{MBN}$, i.e., the tilt angle of the nitrile function $(\theta)$ and thus the angle of the dipole moment of the entire molecule with respect to the surface normal $(\beta)$. One may, therefore, take the approach suggested by Ballav et al. [16] to estimate the angles $\beta$ and $\theta$ starting with $E_{0}=1.277 \mathrm{~V}$, i.e., the experimentally derived value. Under these conditions, a $\beta$ angle of $38^{\circ}$ is predicted, corresponding to a tilt angle $(\theta)$ of $26^{\circ}$, which is in good agreement with those reported for thiophenol on $\mathrm{Ag}[27,28]$.

Either way, it should be noted that previous experimental and theoretical studies on SAM structures and specifically on the tilt angle refer to the SAMs on smooth metals in air or in vacuo. Possible structural differences of the SAMs due to the nanostructured metal surfaces or the contact with an aqueous solution or in an electrochemical environment are not considered. Moreover, the present analysis tacitly assumes that the tilt angle of the nitrile function is potential-independent. The variation in the SEIRA peak intensities in Figure 5 allows one to estimate the underlying error of this assumption. In the ATR SEIRA set-up, the IR signals are enhanced via the electric field component of the electromagnetic radiation perpendicular to the surface $\left|\vec{E}_{\text {rad }}\right|_{\perp}$, such that the maximum enhancement is achieved for molecular oscillators that are oriented in that direction. Since the SEIRA intensity $\mathrm{I}_{\text {SEIRA }}$ scales with the square of $\left|\overrightarrow{\mathrm{E}}_{\text {rad }}\right|_{\perp}$, it follows that $\mathrm{I}_{\text {SEIRA }} \propto(\cos \theta)^{2}$. Assuming that the SEIRA intensity for a pure MBN SAM on an Au electrode, measured at an electrode potential of $+0.1 \mathrm{~V}$, would refer to a tilt angle of the nitrile function with respect to the surface normal of $49^{\circ}$ (vide supra) the increased and decreased intensities at $-0.4 \mathrm{~V}$ and $+0.6 \mathrm{~V}$ then corresponded to a tilt angle of $45^{\circ}$ and $57^{\circ}$, respectively.

\section{Experimental Section}

\subsection{Materials}

6-Mercaptohexanoic acid (MHA), 4-mercaptobenzonitrile $(\mathrm{MBN})$, and thiophenol were purchased from Dojindo, Apin Chemicals, and Sigma-Aldrich, respectively. All other chemicals and solvents 
were of the highest purity grade available. Water was purified by a Millipore system and had a resistivity $>18 \mathrm{M} \Omega \cdot \mathrm{cm}$.

\subsection{SAM Preparation}

Prior to SAM deposition, $\mathrm{Au}$ films and $\mathrm{Ag}$ ring electrodes were prepared and electrochemically roughened as described previously [39,40]. MBN SAMs on Ag were obtained by immersing the electrode in a solution of $1 \mathrm{mM}$ MBN in dimethyl sulfoxide (DMSO): $\mathrm{H}_{2} \mathrm{O}(3: 1 \mathrm{v} / \mathrm{v})$ at $4{ }^{\circ} \mathrm{C}$ for $18 \mathrm{~h}$; for SAM formation on the Au surface, a $1 \mathrm{mM} \mathrm{MBN}$ solution in anhydrous DMSO was employed. Mixed thiophenol/MBN SAMs on Au were prepared in two steps. First, a pure thiophenol SAM was formed from a $2 \mathrm{mM}$ solution of thiophenol in DMSO: $\mathrm{H}_{2} \mathrm{O} 3: 1(\mathrm{v} / \mathrm{v})$, using the same procedure as for MBN SAM formation in the spectro-electrochemical cell. Subsequently, the cell was washed successively with DMSO, ethanol, and water. Afterwards a $10 \mathrm{mM}$ phosphate buffer solution $(\mathrm{pH}=7)$ was added to the cell. Finally, the addition of MBN dissolved in DMSO: $\mathrm{H}_{2} \mathrm{O} 3: 1(\mathrm{v} / \mathrm{v})$ to the cell under potential control $(0 \mathrm{~V} v s$. $\mathrm{Ag} / \mathrm{AgCl})$ resulted in a final concentration of $100 \mathrm{nM}$. In a similar way, a mixed MHA/MBN SAM was formed, starting with the pure MHA SAM [11,39] followed by addition of MBN $(10 \mu \mathrm{M})$ to the solution at $-0.4 \mathrm{~V}$. The formation of the SAMs was monitored by $\mathrm{CV}$ and SEIRA spectroscopy (Supplementary Information).

\subsection{Spectro-Electrochemical Measurements}

SEIRA measurements were carried out in the Kretschmann-ATR configuration which was integrated in an electrochemical cell for potential-controlled studies. Details of the set-up are given elsewhere [39]. SEIRA spectra were recorded with a spectral resolution of $4 \mathrm{~cm}^{-1}$ on a Bruker IFS66v/s spectrometer, using a liquid nitrogen cooled photoconductive MCT detector. 400 scans were co-added for each spectrum; three to five spectra were recorded for each experiment and averaged. Each SEIRA experiment was repeated at least twice.

SER spectroelectrochemical experiments were carried out with the set-up described previously [41]. The spectra were obtained with the 413-nm excitation line of a Krypton ion laser using a confocal Raman spectrometer equipped with a liquid nitrogen cooled CCD detector. The spectral resolution was better than $2 \mathrm{~cm}^{-1}$. The power of the focused laser beam on the sample was $1 \mathrm{~mW}$. The accumulation time was $60 \mathrm{~s}$. All experiments were repeated four times.

CV measurements were carried out in the same cells used for SEIRA and SER spectroelectrochemistry [39,41]. In all electrochemical and spectroelectrochemical measurements potassium phosphate buffer $(10 \mathrm{mM}, \mathrm{pH}=7.0)$ was used. Unless otherwise indicated, all potentials cited in this work refer to the $\mathrm{Ag} / \mathrm{AgCl}$ electrode $(3 \mathrm{M} \mathrm{KCl})$.

\section{Conclusions}

As a prerequisite for exploiting the VSE of MBN monolayers in electrochemical environments, the various factors affecting the stretching frequency of the nitrile function were analyzed and shown to include the effect of (i) MBN binding to the metal and the formation of a SAM, (ii) the surface potential due to the alignment of polar solvent molecules on the SAM surface, (iii) hydrogen bonding 
interactions in aqueous solutions, and finally (iv) the external electric field. The potential dependence of the $\mathrm{C} \equiv \mathrm{N}$ stretching is linear in the potential ranges studied for the various metal/SAM systems, which facilitates the translation of frequency shifts into local electric fields. For this analysis, an electrostatic description is presented that, unlike previous models, takes into account the potential-dependent variation of the charge density in the inner Helmholtz layer of the SAM. For $\mathrm{Au} / \mathrm{MBN}$, the effective potential of zero charge derived from this model is in good agreement with the values calculated from literature data. The more significant deviations in the case of $\mathrm{Ag} / \mathrm{MBN}$ may either be related to the approximations and simplifications in the present model or to the uncertainty in the tilt angle value taken from the literature. The present electric field analysis for mixed MHA/MBN is consistent with previous results for pure MHA, thus indicating that MBN may be used as a reporter group for in situ monitoring of local electric field in SAMs that are used for binding biomolecules such as redox proteins and enzymes $[11,42,43]$.

\section{Acknowledgments}

Financial support by the German Research Foundation (DFG) in the framework of the IRTG 1524 (G.S. \& P.H.), by the Leibniz Institute for Molecular Pharmacology in the framework of the Leibniz Graduate School (J.S.), and the cluster of excellence UniCat (I.Z.) is gratefully acknowledged. D.M. acknowledges the Netherlands Organization for Scientific Research (NWO) grant 722.011.003.

\section{References}

1. Love, J.C.; Estroff, L.A.; Kriebel, J.K.; Nuzzo, R.G.; Whitesides, G.M. Self-assembled monolayers of thiolates on metals as a form of nanotechnology. Chem. Rev. 2005, 105, 1103-1169.

2. Samanta, D.; Sarkar, A. Immobilization of bio-macromolecules on self-assembled monolayers: Methods and sensor applications. Chem. Soc. Rev. 2011, 40, 2567-2592.

3. Mbindyo, J.K.N.; Mallouk, T.E.; Mattzela, J.B.; Kratochvilova, I.; Razavi, B.; Jackson, T.N.; Mayer, T.S. Template synthesis of metal nanowires containing monolayer molecular junctions. J. Am. Chem. Soc. 2002, 124, 4020-4026.

4. Smith, C.P.; White, H.S. Theory of the interfacial potential distribution and reversible voltammetric response of electrodes coated with electroactive molecular films. Anal. Chem. 1992, 64, 2398-2405.

5. Ramírez, P.; Granero, A.; Andreu, R.; Cuest, A.; Mulder, W.H.; Calvente, J.J. Potential of zero charge as a sensitive probe for the titration of ionizable self-assembled monolayers. Electrochem. Commun. 2008, 10, 1548-1550.

6. Oklejas, V.; Sjostrom, C.; Harris, J.M. SERS detection of the vibrational stark effect from nitrile-terminated SAMs to probe electric fields in the diffuse double-layer. J. Am. Chem. Soc. 2002, 124, 2408-2409.

7. Oklejas, V.; Harris, J.M. In-situ investigation of binary-component self-assembled monolayers: A SERS-based spectroelectrochemical study of the effects of monolayer composition on interfacial structure. Langmuir 2003, 19, 5794-5801.

8. Suydam, I.T.; Snow, C.D.; Pande, V.S.; Boxer, S.G. Electric fields at the active site of an enzyme: Direct comparison of experiment with theory. Science 2006, 313, 200-204. 
9. Fafarman, A.T.; Sigala, P.A.; Herschlag, D.; Boxer, S.G. Nitrile bonds as infrared probes of electrostatics in ribonuclease S. J. Am. Chem. Soc. 2010, 132, 12811-12813.

10. Jo, H.; Culik, R.M.; Korendovych, I.V.; DeGrado, W.F.; Gai, F. Selective incorporation of nitrile-based infrared probes into proteins via cysteine alkylation. Biochemistry 2010, 49, 10354-10356.

11. Schkolnik, G.; Utesch, T.; Salewski, J.; Tenger, K.; Millo, D.; Kranich, A.; Zebger, I.; Schulz, C.; Zimányi, L.; Rákhely, G.; et al. Mapping local electric fields in proteins at biomimetic interfaces. Chem. Commun. 2012, 48, 70-72.

12. Aschaffenburg, D.J.; Moog, R.S. Probing hydrogen bonding environments: Solvatochromic effects on the CN vibration of benzonitrile. J. Phys. Chem. B 2009, 113, 12736-12743.

13. Ghosh, A.; Remorino, A.; Tucker, M.J.; Hochstrasser, R.M. 2D IR photon echo spectroscopy reveals hydrogen bond dynamics of aromatic nitriles. Chem. Phys. Lett. 2009, 469, 325-330.

14. Murgida, D.H.; Hildebrandt, P. Electron-transfer processes of cytochrome c at interfaces. New insights by surface-enhanced resonance raman spectroscopy. J. Phys. Chem. B 2001, 105, 1578-1586.

15. Lecomte, S.; Hildebrandt, P.; Soulimane, T. Dynamics of the heterogeneous electron-transfer reaction of cytochrome c552 from thermus thermophilus. A time-resolved surface-enhanced resonance raman spectroscopic study. J. Phys. Chem. B 1999, 103, 10053-10064.

16. Fafarman, A.T.; Sigala, P.A.; Schwans, J.P.; Fenn, T.D.; Herschlag, D.; Boxer, S.G. Quantitative, directional measurement of electric field heterogeneity in the active site of ketosteroid isomerase. Proc. Natl. Acad. Sci. USA 2012, 109, E299-E308.

17. Stein, S.E. Infrared Spectra. In NIST Chemistry WebBook, NIST Standard Reference Database Number 69; Linstrom, P.J., Mallard, W.G., Eds.; National Institute of Standards and Technology: Gaithersburg, MD, USA, 2003. Available online: http://webbook.nist.gov (accessed on 6 May 2012).

18. Suydam, I.T.; Snow, C.D.; Pande, V.S.; Boxer, S.G. Electric fields at the active site of an enzyme: Direct comparison of experiment with theory. Science 2006, 313, 200-204.

19. Natan, A.; Kuritz, N.; Kronik, L. Polarizability, susceptibility, and dielectric constant of nanometer-scale molecular films: A microscopic view. Adv. Funct. Mater. 2010, 20, 2077-2084.

20. Trasatti, S. Work function, electronegativity, and electrochemical behaviour of metals. J. Electroanal. Chem. 1971, 33, 351-378.

21. Valette, G. Hydrophilicity of metal surfaces, silver, gold and copper electrodes. J. Electroanal. Chem. 1982, 139, 285-301.

22. Wang, L.; Rangger, G.M.; Romaner, L.; Heimel, G.; Bučko, T.; Ma, Z.; Li, Q.; Shuai, Z.; Zojer, E. Electronic structure of self-assembled monolayers on $\mathrm{Au}(111)$ surfaces: The impact of backbone polarizability. Adv. Funct. Mater. 2009, 19, 3766-3775.

23. Heimel, G.; Romaner, L.; Zojer, E.; Brédas, J.L. Toward control of the metal-organic interfacial electronic structure in molecular electronics: A first-principles study on self-assembled monolayers of $\pi$-conjugated molecules on noble metals. Nano Lett. 2007, 7, 932-940.

24. Ballav, N.; Schüpbach, B.; Dethloff, O.; Feulner, P.; Terfort, A.; Zharnikov, M. Direct probing molecular twist and tilt in aromatic self-assembled monolayers. J. Am. Chem. Soc. 2007, 129, $15416-15417$. 
25. Frey, S.; Stadler, V.; Heister, K.; Eck, W.; Zharnikov, M.; Grunze, M. Structure of thioaromatic self-assembled monolayers on gold and silver. Langmuir 2001, 17, 2408-2415.

26. Käfer, D.; Bashir, A.; Witte, G. Interplay of anchoring and ordering in aromatic self-assembled monolayers. J. Phys. Chem. C 2007, 111, 10546-10551.

27. Han, S.W.; Lee, S.J.; Kim, K. Self-assembled monolayers of aromatic thiol and selenol on silver: Comparative study of adsorptivity and stability. Langmuir 2001, 17, 6981-6987.

28. Schalnat, M.C.; Pemberton, J.E. Comparison of a fluorinated aryl thiol self-assembled monolayer with its hydrogenated counterpart on polycrystalline Ag substrates. Langmuir 2010, 26, 11862-11869.

29. Shaw, S.K.; Lagutchev, A.; Dlott, D.D.; Gewirth, A.A. Sum-frequency spectroscopy of molecular adsorbates on low-index Ag surfaces: Effects of azimuthal rotation. Anal. Chem. 2009, 81, 1154-1161.

30. Akinaga, Y.; Nakajima, T.; Hirao, K. A density functional study on the adsorption of methanethiolate on the (111) surfaces of noble metals. J. Chem. Phys. 2001, 114, 8555-8564.

31. Ehler, T.T.; Malmberg, N.; Noe, L.J. Characterization of self-assembled alkanethiol monolayers on silver and gold using surface plasmon spectroscopy. J. Phys. Chem. B 1997, 101, 1268-1272.

32. Gershevitz, O.; Sukenik, C.N. In situ FTIR-ATR analysis and titration of carboxylic acid-terminated SAMs. J. Am. Chem. Soc. 2004, 126, 482-483.

33. Burris, S.C.; Zhou, Y.; Maupin, W.A.; Ebelhar, A.J.; Daugherty, M.W. The effect of surface preparation on apparent surface pKa's of $\omega$-mercaptocarboxylic acid self-assembled monolayers on polycrystalline gold. J. Phys. Chem. C 2008, 112, 6811-6815.

34. Emets, V.V.; Damaski, B.B. The relation between the potential of zero charge and work function for sp-metals. Russ. J. Electrochem. 2009, 45, 45-57.

35. Trasatti, S. The concept and physical meaning of absolute electrode potential: A reassessment. J. Electroanal. Chem. 1982, 172, 1-13.

36. Heimel, G.; Romaner, L.; Zojer, E.; Bredas, J.L. The interface energetics of self-assembled monolayers on metals. Acc. Chem. Res. 2008, 41, 721-729.

37. Natan, A.; Kronik, L.; Haick, H.; Tung, R.T. Electrostatic properties of ideal and non-ideal polar organic monolayers: Implications for electronic devices. Adv. Mater. 2007, 19, 4103-4117.

38. Alloway, D.M.; Graham, A.L.; Yang, X.; Mudalige, A.; Colorado, R., Jr.; Wysocki, V.H.; Pemberton, J.E.; Lee, T.R.; Wysocki, R.J.; Armstrong, N.R. Tuning the effective work function of gold and silver using $\omega$-functionalized alkanethiols: Varying surface composition through dilution and choice of terminal groups. J. Phys. Chem. C 2009, 113, 20328-20334.

39. Wisitruangsakul, N.; Zebger, I.; Ly, K.H.; Murgida, D.H.; Egkasit, S.; Hildebrandt, P. Redox-linked protein dynamics of cytochrome $\mathrm{C}$ probed by time-resolved surface enhanced infrared absorption spectroscopy. Phys. Chem. Chem. Phys. 2008, 10, 5276-5286.

40. Wackerbarth, H.; Klar, U.; Gunther, W.; Hildebrandt, P. Novel time-resolved surface-enhanced (resonance) raman spectroscopic technique for studying the dynamics of interfacial processes: Application to the electron transfer reaction of cytochrome c at a silver electrode. Appl. Spectrosc. 1999, 53, 283-291. 
41. Ly, K.H.; Wisitruangsakul, N.; Sezer, M.; Feng, J.J.; Kranich, A.; Weidinger, I.; Zebger, I.; Murgida, D.H.; Hildebrandt, P. Electric field effects on the interfacial electron transfer and protein dynamics of cytochrome c. J. Electroanal. Chem.2011, 660, 367-376.

42. Murgida, D.H.; Hildebrandt, P. Redox and redox-coupled processes of heme proteins and enzymes at electrochemical interfaces. Phys. Chem. Chem. Phys. 2005, 7, 3773-3784.

43. Ly, H.K.; Sezer, M.; Wisitruangsakul, N.; Feng, J.J.; Kranich, A.; Millo, D.; Weidinger, I.M.; Zebger, I.; Murgida, D.H.; Hildebrandt, P. Surface-enhanced vibrational spectroscopy for probing transient interactions of proteins with biomimetic interfaces: Electric field effects on structure, dynamics and function of cytochrome c. FEBS J. 2011, 278, 1382-1390.

(C) 2012 by the authors; licensee MDPI, Basel, Switzerland. This article is an open access article distributed under the terms and conditions of the Creative Commons Attribution license (http://creativecommons.org/licenses/by/3.0/). 